Revue d'histoire de l'Amérique française

REVUE D.HISTOIRE DE L'AMÉRIQUE FRANÇAISE

\title{
Un siècle de vie française en dehors du Québec
}

\section{Richard Arès}

Volume 21, numéro 3a, 1967

Cent ans d'histoire 1867-1967

URI : https://id.erudit.org/iderudit/302712ar

DOI : https://doi.org/10.7202/302712ar

Aller au sommaire du numéro

Éditeur(s)

Institut d'histoire de l'Amérique française

ISSN

0035-2357 (imprimé)

1492-1383 (numérique)

Découvrir la revue

Citer cet article

Arès, R. (1967). Un siècle de vie française en dehors du Québec. Revue d'histoire de l'Amérique française, 21(3a), 531-570. https://doi.org/10.7202/302712ar d'utilisation que vous pouvez consulter en ligne.

https://apropos.erudit.org/fr/usagers/politique-dutilisation/ 
Richard Arès

\section{UN SIECLE}

DE VIE FRANÇAISE

EN DEHORS

DU QUEBEC 


\section{Richard Arès}

Né à Marieville, comté de Rouville, le 7 janvier 1910.

Etudes au Séminaire de Saint-Hyacinthe, au Scolasticat des Jésuites, à l'Université de Montréal (M.A., L. ès Sc. Doc. Economiques et Politiques), à l'Institut catholique de Paris (D.Ph., D. Sc. Soc.), à l'Université de Paris (diplôme d'études supérieures et docìorat en droit international), à l'Institut d'Etudes politiques, Université de Paris (sciences politiques).

Ordonné prêtre le 13 août 1944.

Professeur de philosophie au Collège de St-Boniface, Manitoba (1940-1941); Collège Jean-de-Brébeuf, Montréal (1946-47).

Professeur de doctrine sociale de l'Eglise à l'Université de Montréal (1951-1952).

Membre I.S.P.

Directeur de Relations; membre de la Commission royale d'enquêtè sur les problèmes constitutionnels (1953-1956).

Directeur de la Ligue d'Action nationale. Président des Semaines sociales du Canada (secteur français, depuis 1958). Membre de la Société Royale du Canada et de l'Académie des Sciences morales et politiques du Québec.

\section{Oeuvres :}

Petit catéchisme anticommuniste (1937)

Catéchisme de l'organisation corporative (1938)

Petit catéchisme d'éducation syndicale (1938)

Dossier sur le pacte fédératif de $1867(1941,1967)$

Nos positions françaises au Manitoba (1941)

Petit guide moral du législateur (1943)

L'Eglise et le nationalisme (1944)

Notre question nationale $-\mathrm{t}$. I: Les faits; II: Positions de principe;

III: Positions patriotiques et nationales (1947)

L'Eglise catholique et l'organisation de la société internationale contemporaine (1949)

La Confédération: Pacte ou Loi ? (1949)

Essais de réforme de l'entreprise aux Etats-Unis (1951)

Vers la corporation agricole (en coll. avec M. Clément) (1951)

Le fédéralisme (1952)

Qu'est-ce que la sécurité sociale? (1953)

Capitalisme, syndicalisme et organisation professionnelle (1953)

Positions du français au Canada (1954)

Pie XII et l'Ordre politique (1956)

Les Caisses populaires et la communauté canadienne-française (1961) Pour un Québec fort (1962)

Du rôle de l'Etat dans un Québec fort (1962)

Justice et équité pour la communauté canadienne-française (1963)

Quand tout le Québec parlera français! (en collaboration) (1963)

Conditions de survie de la langue et de la culture françaises au Manitoba (1964)

Le devenir politique du Québec (en collaboration) (1965) 


\section{UN SIECLE DE VIE FRANÇAISE EN DEHORS DU QUÉBEC}

Pas plus que l'histoire du Canada, l'histoire de la vie française en dehors du Québec ne commence en 1867. Bien avant cette date, des groupes français existaient ou étaient en train de se constituer dans les Maritimes, en Ontario et dans les vastes et lointains territoires de l'Ouest. A ces groupes jusque-là épars, la Confédération va apporter une certaine unité politique en même temps que des moyens de communication et un sens nouveau de solidarité. À mesure que le Canada s'agrandit, le Canada français lui-même prend de l'ampleur, déborde les provinces centrales, s'annexe les groupements français des Maritimes et de l'Ouest canadien et finit par s'étendre, lui aussi, d'un Océan à l'autre.

L'objet du présent article est de présenter l'évolution de la vie française en dehors du Québec depuis la Confédération. Tâche immense et hérissée de difficultés, car toutes les provinces ne sont pas entrées à la même date dans l'Union ni aux mêmes conditions pour la vie française. De plus, à quoi mesurer la qualité de celle-ci ? Au nombre de Canadiens d'origine française ou à celui de Canadiens de langue maternelle française ? Aux difficultés affrontées et surmontées ? Aux institutions mises en place et encore en vigueur dans chacune des provinces? Pour répondre adéquatement à ces questions, il faudrait procéder à de longues enquêtes, dont les résultats, publiés, prendraient aisément les dimensions d'un gros volume. Mon étude sera beaucoup plus modeste; elle comportera d'abord un coup d'œil sur les garanties offertes à la vie française par la constitution de 1867, puis un aperçu de l'évolution globale de cette vie française en dehors du Québec et enfin quelques données plus particulières concernant la situation dans chaque province. 


\section{I - LES GARANTIES CONSTITUTIONNELLES DONNEES AUX FRANCO-CANADIENS}

Pour simple raison de commodité, c'est-à-dire pour éviter d'employer constamment les expressions du genre de "nos minorités françaises" ou de "les Canadiens français d'en dehors du Québec", j'emploie ici les mots Franco-Canadiens précisément pour désigner tous les Canadiens d'expression française vivant dans une province autre que celle de Québec. Quelles garanties pour leur vie française ces Franco-Canadiens possèdent-ils en vertu de la Constitution de 1867 ? En réponse, on met habituellement de l'avant deux articles de l'Acte de l'Amérique du Nord britannique: l'article 133 et l'article 93 , mais que vaut la protection offerte par ces deux articles?

\section{L'Article 133}

Des 147 articles que comporte l'Acte de l'Amérique du Nord britannique, un seul concerne la question des langues au Canada: l'article 133, lequel établit le bilinguisme au niveau fédéral et au niveau provincial québécois à la fois dans les Chambres et devant les tribunaux ${ }^{1}$. Il s'agit done essentiellement d'un bilinguisme politique et judiciaire, non directement d'un bilinguisme scolaire. Malheureusement, c'est autour et à propos de l'école que les Franco-Canadiens auront à livrer la bataille la plus acharnée pour leur survivance. Est-ce à dire que l'article 133 ne leur sera d'aucune utilité et ne leur accordera aucune protection ? Oui, tant que l'on s'en tiendra à une interprétation strictement littérale et légale de cet article; non, chaque fois que l'on consentira à dépasser les vues de la stricte légalité pour s'engager sur les voies de l'équité, du fairplay et de la générosité.

\footnotetext{
1 Texte de l'article 133: "Dans les chambres du parlement du Canada et de la législature du Québec, chacun pourra, dans les débats, faire usage de la langue anglaise ou de la langue française; mais les registres et les procès-verbaux des chambres susdites devront être tenus dans ces deux langues. Dans tout procès porté devant un tribunal du Canada, établi en vertu de la présente loi ou devant un tribunal du Québec, chacun pourra faire usage de l'une ou de l'autre de ces langues dans les procédures et les plaidoyers qui y seront faits ou dans les actes de procédure qui en émaneront.

Les lois du parlement du Canada et de la législature du Québec devront être imprimées et publiées dans l'une et l'autre de ces langues."
} 
Au cours du siècle qui vient de s'écouler, les Franco-Canadiens - aidés en cette matière par des Franco-Québécois comme Henri Bourassa - développeront à partir de l'article 133, deux arguments tendant à démontrer le droit du français à exister et à s'épanouir par tout le Canada. Le premier en sera une conclusion indirecte, le second s'appuiera sur le sens profond à donner à cet article.

Dans leur volonté de faire reconnaître les droits de leur langue, les Franco-Canadiens développeront d'abord l'argument suivant: l'article 133 reconnaît l'égalité des langues au niveau fédéral; mais comment les Franco-Canadiens pourront-ils exercer ce droit au français, s'ils ne peuvent apprendre leur langue dans les écoles de leur province ? A quoi sert cette garantie fédérale si des dispositions provinciales peuvent la rendre inopérante ? Toute sa vie, dans ses nombreux discours consacrés à la défense des minorités françaises: du Nord-Ouest, du Keewatin, de la Saskatchewan, de l'Alberta et de l'Ontario, Henri Bourassa a pétri et ressassé cet argument. Voici, par exemple, comment il le développait dans sa défense de la minorité franco-ontarienne:

L'article 133 ... reconnaît l'égalité des deux langues dans tous les domaines du gouvernement et de l'administration nationale. Tout député canadien-français de l'Ontario ou du Nouveau-Brunswick, du Québec ou de l'Alberta, a le droit de parler sa langue au parlement. Tout contribuable canadien-francais de Toronto ou de Montréal, de Winnipeg, de Halifax ou de Vancouver, a le droit d'exiger que tous les documents de l'Etat soient imprimés dans sa langue. Il a le droit de parler et d'écrire en français à chacun des représentants de l'administration fédérale.

Tout justiciable canadien-français, dans chacune des provinces et jusqu'aux confins de la mer Glaciale, dans le territoire du Yukon et celui du Mackenzie, a le droit de plaider ou de témoigner en français devant tous les tribunaux fédéraux.

Ces droits sont acquis à toutes les générations de Canadiens-français qui se succèderont dans toutes les parties de la Confédération canadienne. Or, je vous le demande, comment les générations futures pourront-elles jouir de ces droits et les exercer dans 
leur plénitude, si les autorités de l'une quelconque des provinces empêchent systématiquement les enfants de la génération actuelle d'acquérir à l'école la connaissance parfaite de leur langue? N'est-ce pas tarir la source même du droit ? ${ }^{2}$

Dans leur mémoire à la Commission Rowell-Sirois, en 1938, les Acadiens et les Canadiens français des provinces Maritimes reprenaient cet argument et en défendaient avec force la validité. L'article 133, disaient-ils, est incomplet. Il définit certains droits du français, mais ne dit rien de l'école.

Mais, pour des gens de bonne foi, le bilinguisme scolaire ne découle-t-il pas de l'article 133 comme conséquence logique et inéluctable? Limiter à l'enceinte du parlement ou des tribunaux fédéraux l'emploi de la langue française est une dérision et une tromperie. C'est répudier la convention de 1867, c'est biffer un texte dont le but fut de maintenir le français comme l'une des deux langues officielles du Canada, de laisser chaque Canadien libre de l'enseigner ou de l'apprendre, de l'écrire ou de le parler. Quelle folie eût été celle des "Pères" en décrétant cet usage du français au parlement et devant les tribunaux fédéraux, si leur intention n'avait pas été de rendre possible partout au Canada l'emploi du français? Si l'on a le droit de plaider et de témoigner en français devant les tribunaux fédéraux, si l'on a le droit de parler français au parlement, si l'on a le droit de lire en français tous les documents fédéraux et de correspondre ou de causer en français avec chacun des représentants de l'administration fédérale, ne s'ensuit-il pas que l'idée mère de la loi organique de 1867 est de mettre le français partout au Canada sur un pied d'égalité avec l'anglais?

(...) Malheureusement, les provinces anglaises n'ont pas encore donné à l'article 133 cette interprétation large et rationnelle. Dans les Provinces Maritimes, la majorité a imposé à la minorité un programme scolaire pratiquement tout anglais, et qui ne convient nullement à des enfants de langue française qui ne

\footnotetext{
2 Henri Bourassa, La langue française au Canada: ses droits, sa nécessité, ses avantages (Montréal, 1915), 30.
} 


\section{connaissent pas la langue anglaise à leur entrée à} l'école...

J'ai tenu à citer ces deux dernières phrases, précisément pour montrer que l'argumentation franco-canadienne fondée sur une interprétation large de l'article 133 n'était guère acceptée en dehors du Québec, surtout quand il s'agissait de reconnaître aux minorités françaises le droit à des écoles de leur langue.

Il faut ajouter que les Franco-Canadiens n'eurent guère plus de succès auprès de leurs compatriotes anglo-canadiens quand ils tentèrent de donner à la Confédération, en particulier à l'article 133, le sens profond d'une association dans l'égalité entre les deux principales nationalités existant en 1867. Pour eux, la Confédération ne pouvait avoir d'autre sens. John A. Macdonald avait en 1865 nettement déclaré: "Les délégués de toutes les provinces ont consenti à ce que l'usage de la langue française formât l'un des principes sur lesquels serait basée la Confédération." 4 Plus tar:d, premier ministre du Canada, le même Macdonald avait précisé le sens profond de la constitution canadienne. Refusant de s'associer à la campagne pour faire abolir les droits du français dans les territoires du Nord-Ouest, il avait dit: "Nous avons une constitution en vertu de laquelle tous les sujets anglais sont sur un pied d'égalité, ayant des droits égaux en matière de langue...." 5

S'inspirant de ces paroles, Henri Bourassa ne cessa d'enseigner dans ses discours que la Confédération se définissait et devait continuer à se définir comme étant "la libre et volontaire association de deux peuples, jouissant de droits égaux en toutes matières" ${ }^{6}$. Doctrine qui devait recevoir une éclatante confirmation lors de la création de la Commission royale d'enquête sur le bilinguisme et le biculturalisme: le mandat de celle-ci lui enjoignait, en effet, de rechercher "les mesures à prendre pour

\footnotetext{
3"Mémoire des Acadiens et des Canadiens français des Maritimes", dans Mémoires des minorités catholiques de langue francaise au Canada présentés à la Commission Rowell (Montréal, juin 1938), L'Oeuvre des Tracts, no 228, 2-3.

4 Débats sur la Confédération, 10 mars 1865, 943.

5 Débats de la Chambre des Communes, 17 février 1890, 764.

6 La Conscription (Montréal, 1917), 20.
} 
que la Confédération canadienne se développe d'après le principe de l'égalité des deux peuples qui l'ont fondée".

Le malheur a été, cependant, que cette doctrine a été plus affirmée sur le papier que vécue dans la réalité. Alors que les Canadiens français interprétaient ainsi la Confédération, les Anglo-Canadiens, pour la plupart, l'interprétaient comme créant un Canada unilingue au sein duquel serait toléré un Québec unilingue. Dans son discours du 17 décembre 1962 à la Chambre des communes, alors qu'il était chef de l'opposition, M. Lester Pearson a clairement exprimé ce malentendu fondamental sur le sens à donner à la Confédération et, en définitive, à l'article 133:

La Confédération ne constitue peut-être pas un traité, à proprement parler, ni un pacte entre Etats. Mais il s'agissait d'une entente pour arriver à un règlement entre les deux races fondatrices $\mathrm{du} \mathrm{Ca}$ nada, sur la base d'une association acceptable entre égaux. Ce règlement prévoyait la réalisation et le maintien de l'unité nationale et politique sans qu'on impose au pays l'uniformité du point de vue racial, culturel ou linguistique.

Je pense parfois que cette entente était plus théorique que réelle. En dehors du Québec, à mesure que le Canada se développait d'un océan à l'autre, cette entente était plus souvent marquée par des infractions que par son observance, et cela pour des raisons que chacun d'entre nous qui connaît l'évolution du Canada peut comprendre. Il en est résulté deux différentes interprétations de la Confédération. Et c'est cette différence d'interprétation du concept même de la Confédération qui a été et qui est encore aujourd'hui une source de confusion, de frustration et même de conflit.

Pour les Canadiens de langue française, la Confédération créait une nation bilingue et biculturelle. Elle protégeait leur langue et leur culture dans tout le Canada. Elle signifiait une association, et non pas une domination. Les Canadiens francophones ont cru que cette association signifiait des chances et des possibilités égales pour les deux races fondatrices, à toutes les étapes de la croissance du pays. 
Les Canadiens de langue anglaise reconnaissent, bien sûr, que l'entente confédérative protégeait les droits des Canadiens français dans la province de Québec, au Parlement et dans les tribunaux fédéraux. Mais la plupart avaient l'impression, et je ne crois pas me tromper en disant cela, qu'elle ne s'étendait pas plus loin que ces limites, du moins jusqu'à une époque encore très récente. Cela signifiait qu'à toutes fỉns utiles, il existerait un Canada de langue anglaise, avec un Québec bilingue, ce qu'on appelle le "fait français" devait être quelque chose d'uniquement provincial ?

Voilà un texte capital et lumineux. Durant le siècle qui vient de s'écouler, deux conceptions de la Confédération se sont affrontées, et celle qui a prévalu, dans neuf provinces sur dix, du moins jusqu'à ces derniers temps, a été celle de la majorité anglo-canadienne, c'est-à-dire celle d'un Canada de langue anglaise comportant une "réserve" bilingue, le Québec. Cela étant, il n'est pas difficile de comprendre pourquoi le "siècle de vie française en dehors du Québec" se résume en cent ans de luttes pour la survivance, en cent ans de luttes de la part des FrancoCanadiens pour se faire reconnaître le droit à l'existence comme groupe national dans les autres provinces que le Québec. Encore aujourd'hui, la plupart de leurs compatriotes anglo-canadiens contestent l'interprétation qu'ils donnent de l'article 133.

\section{L'article 93}

Si encore pour se consoler de leurs déboires, les FrancoCanadiens avaient pu compter sur l'article 93, ayant trait à l'enseignement et à l'école et l'invoquer en faveur, non seulement de leur religion, mais aussi de leur langue; là aussi, ils ne rencontrèrent qu'incompréhension et intolérance. D'après cet article, comme on le sait, la juridiction sur l'enseignement appartient aux provinces, celles-ci cependant ne pouvant porter atteinte aux droits et privilèges des écoles confessionnelles. La langue fait-elle partie de ces droits et privilèges ? Les Franco-Ontariens, en particulier, essayèrent de faire admettre ce point de vue, mais les tribunaux refusèrent de leur donner raison.

7 Débats de la Chambre des Communes, 17 décembre 1962, 2858-2860. 
Le cas vaut d'être signalé un peu plus longuement. A la suite du Règlement 17 de 1913 restreignant en Ontario l'usage du français dans les écoles, les commissaires catholiques canadiens-français d'Ottawa plaidèrent que le libre usage du français dans les écoles séparées était un droit protégé par la première clause de l'article 93 et qu'en conséquence la législature de l'Ontario ne pouvait l'abroger. Aux yeux des Franco-Ontariens, ce droit était d'autant plus fort que le français possédait le statut de langue officielle au Canada, et en particulier en Ontario depuis l'Acte de Québec de 1774.

Parlant en 1915 de cette question, Henri Bourassa déclarait:

L'hon. Belcourt a démontré devant les tribunaux que les garanties offertes par l'Article 93 ne s'appliquent pas seulement à l'existence des écoles séparées mais à tous les privilèges relatifs à ces écoles. Il a prouvé que, dans la plupart des écoles séparées fondées par les Canadiens-français du Haut-Canada, la langue française était enseignée sous l'autorité des lois et des règlements scolaires de la province. Il en conclut que cet enseignement constitue l'un des privilèges relatifs à l'école séparée et garantis par la constitution. C'était également l'opinion de sir Richard Scott, auteur de la loi des écoles séparées du Haut-Canada.

Nul doute, comme l'a fort bien dit l'hon. M. David au Sénat, que Cartier n'eût jamais consenti à signer le pacte fédéral s'il n'avait été convaincu de la valeur de cette garantie ${ }^{8}$.

Bourassa se montrait trop optimiste. La cause gravit tous les échelons jusqu'au Comité judiciaire du Conseil privé, lequel confirma la sentence des tribunaux inférieurs. En réponse au plaidoyer du sénateur Belcourt, le Conseil privé déclara que seuls les droits religieux étaient garantis par l'article 93, non les droits linguistiques, et que, d'un autre côté, dans l'article 133 garantissant ces derniers droits, il n'était aucunement question de l'école ${ }^{9}$.

${ }^{8}$ Henri Bourassa, La langue française au Canada (Montréal, 1915), 29.

$\theta$ Trustees of the Roman Catholic Separate Schools for the City of Ottawa v. Mackell and others (1917), A.C. 62. "Mr. Belcourt urged that so to regulate use of the French language in the separate Roman Catholic 
En conséquence, l'interprétation large et généreuse que les Franco-Ontariens avaient voulu donner de l'article 93 dut s'effacer devant une interprétation étroitement littérale et strictement légale, et les écoles des minorités françaises se trouvèrent dépourvues de toute protection juridique et soumises au régime de la tolérance, laquelle fut loin de leur être toujours accordée. "Bouclier de luxe" pour la minorité protestante du Québec, l'article 93 n'aura été, selon le mot de l'abbé Groulx, pour les minorités franco-catholiques des autres provinces “qu'un paravent de carton" 10.

Si l'expérience d'un siècle de vie française en dehors du Québec démontre quelque chose, c'est bien ceci: les garanties accordées aux Franco-Canadiens par la Constitution de 1867 ont été tout à fait insuffisantes. Les Pères canadiens-français de la Confédération ont manqué de clairvoyance et de fermeté; ils n'ont pas suffisamment tenu compte des groupes français des autres provinces et les ont livrés sans défense aux attaques des fanatiques de l'impérialisme assimilateur ${ }^{11}$.

schools in Ottawa constituted an interference and is in some way inconsistent with a natural right vested in the French-speaking population; but unless this right was one of those reserved by the Act of 1867, such interference could not be resisted, and their Lordships have already expressed the view that people joined together by the union of language and not by the ties of faith do not form a class of persons within the meaning of the Act... In this connection it is worthy of notice that the only section in the British North America Act, 1867, which relates to the use of the English and French languages (s. 133) does not relate to education, and is directed to an entirely different subject-matter..."

${ }^{10}$ Lionel Groulx, L'enseignement français au Canada, I - Dans le Québec (Montréal, 1931), 277.

11 Après coup, après un siècle d'expérience, on ne peut qu'admirer la justesse des paroles prononcées, lors des débats de 1865, par le député Félix Geoffrion, précisément à propos des garanties accordées au français dans les Résolutions de Québec. Répondant au solliciteur général Langevin, qui venait de vanter l'ampleur de ces garanties, le député Geoffrion en montra la faiblesse en ces termes: "Pour ma part, j'avoue que je ne puis comme lui apercevoir cette magnifique protection qu'il nous a vantée. Si les résolutions maintenant devant la chambre veulent dire quelque chose, on ne peut trouver cette signification que dans la lettre même de ces résolutions. Il sera toujours loisible à une majorité anglaise de se servir de la lettre de la constitution et de venir nous dire: cela ne sera pas; nous ne le voulons pas, et la constitution ne vous garantit pas les droits que vous prétendez qu'elle vous confère. Et elle pourra d'autant plus facilement le faire que les résolutions n'affirment pas que ces choses seront inattaquables... Les résolutions devront être interprétées telles qu'elles sont, sans égard à l'intention des conférendaires, 


\section{II - EVOLUTION DEMOGRAPHIQUE GLOBALE}

En dépit des difficultés qu'ils ont rencontrées et des persécutions qu'ils ont subies durant le siècle qui a suivi la Confédération, les Franco-Canadiens n'en ont pas moins réussi à augmenter considérablement leurs effectifs. Combien étaient-ils au moment de la Confédération ? Il n'est pas facile de le dire avec précision pour deux raisons: le premier recensement n'a eu lieu qu'en 1871 et il s'est borné aux quatre provinces originelles du Québec, de l'Ontario, du Nouveau-Brunswick et de la NouvelleÉcosse; de plus, les statistiques qu'il fournit ne valent vraiment que pour les Canadiens d'origine française, non pour les Canadiens de langue maternelle française (pour des raisons techniques qu'il serait trop long d'expliquer ici).

Si l'on s'en tient donc aux effectifs des Canadiens d'origine française, voici comment ils se présentent en 1871.

\section{TABLEAU 1}

Nombre de Canadiens d'origine française, par province, en 1871

Ontario 75,383

Nouveau-Brunswick ... $\ldots \ldots \ldots \ldots \ldots . \quad 44,907$

Nouvelle-Ecosse 32,833

Québec

$$
\begin{array}{r}
153,123 \text { ou } 14.2 \% \\
\frac{929,817}{1,00} 85.8 \%
\end{array}
$$

Canada de 1871

et à cause de cela, je ne puis m'empêcher de déclarer que les CanadiensFrançais commettraient une bien inexcusable imprudence... Et si nous nous contentons de l'entente dont nous a parlé l'hon. solliciteur général du BasCanada, on pourra nous répondre, quand nous voudrons nous opposer à cette injustice: "Il fallait prendre et exiger de plus amples garanties, et vous deviez voir à ce que la constitution fût plus explicite et plus précise sur ce point." Et nous n'aurons rien à répondre à cela! Il faudra nous résigner; il nous faudra subir toutes les restrictions que la majorité pourra nous imposer. Je maintiens donc que la députation canadienne-française de cette chambre devrait exiger du gouvernement que cette entente entre les conférendaires fît partie de la constitution, et que les garanties qu'on nous dit être octroyées par la constitution fussent plus explicitement exprimées qu'elles ne le sont dans les résolutions. Si nous votons ces résolutions telles qu'elles sont, nous voterons sans savoir exactement quelle est la nature des garanties qu'elles nous offrent." (Débats sur la Confédóration (1865), 786787). 
Ainsi, à cette époque, il existe en dehors du Québec un peu plus de 150,000 Canadiens d'origine française. Mais le Canada d'aujourd'hui est beaucoup plus vaste que celui de 1871; il comprend aussi les provinces de l'Ile-du-Prince-Édouard et de TerreNeuve, où vivaient, au temps de la Confédération, une dizaine de mille Acadiens et Canadiens français, et les quatre provinces de l'Ouest canadien, lesquelles devaient renfermer, vers 1870, environ une douzaine de mille Franco-Canadiens. Ce qui veut dire qu'il y a un siècle, il devait y avoir en dehors du Québec dans tous les territoires formant aujourd'hui le pays canadien environ 175,000 Canadiens d'origine française, soit $16 \mathrm{p}$. cent de toute la population canadienne-française de l'époque.

Comment cette population a-t-elle évolué durant le siècle qui vient de s'écouler? Voici un tableau qui nous l'indique.

\section{TABLEAU 2}

Evolution de la population canadienne-française au Québec et en dehors du Québec, de 1871 à 1961

\begin{tabular}{|c|c|c|c|c|}
\hline Innée & u Québec & $\%$ & Hors du Québec & $\%$ \\
\hline 1871 & 929,817 & 858 & 100 & 1 \\
\hline 8 & 1,0 & & & \\
\hline 1901 & 1,32 & & & 10 \\
\hline 191 & 1,60 & $\ldots \ldots$. & & 22.1 \\
\hline 1921 & 1,88 & .1 & & 22.9 \\
\hline 931 & 2,27 & ... 77.5 & & 22.3 \\
\hline$\ldots$ & $2,695,032$ & $\begin{array}{llll}\ldots & \ldots & \ldots & 77.4\end{array}$ & 06 & 22.6 \\
\hline $1 \ldots$ & $3,327,128$ & $\begin{array}{ccccc}\ldots & \ldots & \ldots & \ldots & \mathbf{7 7}\end{array}$ & $992,039 \ldots$ & 23.0 \\
\hline 961 . & $4,241,354$ & $\ldots \ldots \ldots 76.6$ & $1,298,992 \ldots$ & 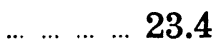 \\
\hline
\end{tabular}

Se chiffrant à 153,123 en 1871 , les effectifs franco-canadiens s'élèvent, en 1961, à 1,298,992, c'est-à-dire qu'ils se sont multipliés par huit, alors que les effectifs franco-québécois passaient de 929,817 à $4,24: 1,354$, ne se multipliant toutefois que par quatre et demi, et c'est bien là le fait étonnant: les Canadiens français ont proportionnellement augmenté leurs effectifs plus en dehors du Québec qu'au Québec même. La conséquence a été 
qu'il s'est opéré, au cours du siècle écoulé, un transfert d'environ 10 p. cent de la population canadienne-française: le Québec qui, au moment de la Confédération, renfermait en ses frontières 85 p. cent de cette population n'en contient plus aujourd'hui que 76 p. cent, et les minorités françaises ont vu leur pourcentage passer de 14 à 23.

Ces pourcentages ne valent cependant que si l'on se place sur le plan de l'origine ethnique, car sur le plan de la langue maternelle la situation n'est plus du tout la même. Or, quand on parle de vie française, c'est la langue, bien plus que l'origine, qui est visée. Le tableau précédent ne nous donne pas, par conséquent, un juste aperçu de l'évolution de la vie française en dehors du Québec. Aussi nous faut-il recourir aux statistiques fondées sur la langue maternelle, c'est-à-dire sur le français en tant qu'il est la langue maternelle des Canadiens, non seulement d'origine française, mais de toutes autres origines.

\section{TABLEAU 3}

Evolution de la population canadienne de langue maternelle française, de 1931 à 1961

\begin{tabular}{|c|c|c|}
\hline Année & Au Québec & Hors du Québec \\
\hline 1931 & $2,292,193$ & 540,705 \\
\hline 1941 & $2,717,287$ & 637,466 \\
\hline 1951 & $3,347,030$ & 721,820 \\
\hline 1961 & $4,269,689$ & 853,462 \\
\hline
\end{tabular}

La marche de l'évolution est ici en sens inverse de celle du tableau précédent: le pourcentage détenu par le Québec va sans cesse augmentant, et celui des autres provinces sans cesse diminuant. L'explication de ce phénomène doit être cherchée dans les nombreuses pertes subies par les Franco-Canadiens sur le plan de leur langue maternelle. Ainsi la distance qui sépare le nombre des Canadiens d'origine française et le nombre de Canadiens de langue maternelle française se révèle plus considérable tous les dix ans. 
TABLEAU 4

Différence entre les effectifs selon l'origine et ceux selon la langue chez les Canadiens français d'en dehors du Québec

\begin{tabular}{|c|c|c|c|}
\hline 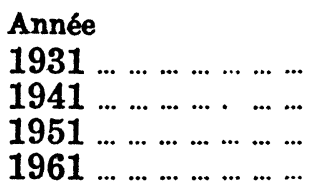 & $\begin{array}{c}\text { Selon l'origine } \\
657,931 \\
788,006 \\
992,039 \\
1,298,992\end{array}$ & $\begin{array}{c}\text { Selon la langue } \\
540,705 \\
637,466 \\
721,820 \\
853,462\end{array}$ & $\begin{array}{c}\text { Différence } \\
117,226 \\
150,540 \\
270,219 \\
445,530\end{array}$ \\
\hline
\end{tabular}

Le fait frappant, ici, est l'augmentation rapide de la différence entre les effectifs selon l'origine et ceux selon la langue au cours des derniers recensements. Pour mieux faire saisir l'ampleur de ce phénomène, reprenons ce même tableau sous une autre forme.

\section{TABLEAU 5}

Augmentation de la différence entre les effectifs selon l'origine et ceux selon la langue chez les Canadiens français d'en dehors du Québec

$\begin{array}{llccc}\text { Années } & & \text { Selon l'origine } & \text { Selon la langue } & \begin{array}{c}\text { Différence } \\ \text { d'augmentation }\end{array} \\ \text { De } 1931 \text { à } 1941 & 130,075 & 96,761 & 33,314 \\ \text { De } 1941 \text { à } 1951 & 204,033 & 84,354 & 119,679 \\ \text { De } 1951 \text { à } 1961 & 306,953 & 131,642 & 175,311\end{array}$

De 1931 à 1941, les effectifs franco-canadiens, comptés selon l'origine, ont augmenté de 130,075, mais, dénombrés selon la langue, ils n'ont augmenté que de 96,761; de 1941 à 1951, cette double augmentation a été, d'une part, de 204,033, et d'autre part de 84,354 seulement; de 1951 à 1961, elle a été de 306,953 sur le plan de l'origine ethnique, mais seulement de 131,642 sur le plan de la langue maternelle française, c'est-à-dire moins de la moitié de l'augmentation selon l'origine.

Ainsi donc les 175,000 Franco-Canadiens de l'époque de la Confédération sont devenus aujourd'hui plus d'un million en dehors du Québec, mais ils n'ont malheureusement pas tous conservé leur langue et ne peuvent pas tous se rattacher à la vie française. Voici comment peut se résumer leur situation linguistique en 1961. 
TABLEAU 6

Bilan de la situation linguistique des Franco-Canadiens, ou Canadiens d'origine française en dehors du Québec, en 1961

D'origine française $\ldots \ldots \ldots \ldots \ldots$

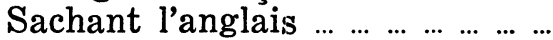

Sachant le français

Nombre

De langue maternelle française ...

L'anglais et le français

$1,298,992$

$1,071,752$

852,429

800,699

629,501

$\begin{array}{ll}\text { De langue maternelle anglaise .... } & 484,400 \\ \text { Ne sachant que l'anglais ........... } & 442,251\end{array}$

Ne sachant que le français

222,658

Pourcentage

$\%$

100.0

82.4

65.6

61.6

48.4

37.3

34.0

17.1

Ces chiffres montrent éloquemment la difficulté de rester français en dehors du Québec. Les Franco-Canadiens savent plus l'anglais que le français; ils comptent 484,400 des leurs dont la langue maternelle est l'anglais et 442,251 qui ne savent plus que l'anglais. Plus de $60 \mathrm{p}$. cent d'entre eux, par contre, peuvent encore parler le français et ont déclaré cette langue leur langue maternelle. Ce sont ces 800,000 Franco-Canadiens qui sont vraiment responsables de la vie française en dehors du Québec, en ce sens qu'ils la portent en eux, lui font place dans leur foyer et la font rayonner autour d'eux dans les écoles, les paroisses et les différentes institutions culturelles, sociales et économiques qu'ils ont bâties pour eux, pour y vivre leur vie française. $\bar{A}$ force de pressions et de pétitions, ils ont réussi à obtenir de Radio-Canada quelques postes de radio et de télévision dans leur langue, mais cela avec quel retard et avec quelle parcimonie en regard du traitement rapide et généreux octroyé à la minorité anglaise du Québec !

\section{III - LA SITUATION DES FRANCO.CANADIENS PAR REGION ET PAR PROVINCE}

On peut diviser les Franco-Canadiens en trois groupes distincts: celui des provinces Maritimes, celui de l'Ontario et celui de l'Ouest canadien. Pour aider le lecteur à se faire tout de suite une idée de l'importance de chacun de ces groupes, je présente un tableau indiquant le nombre et le pourcentage de 
leurs effectifs, tant selon l'origine ethnique que selon la langue maternelle.

\section{TABLEAU 7}

Importance des groupes régionaux franco-canadiens hors du Québec, en 1961

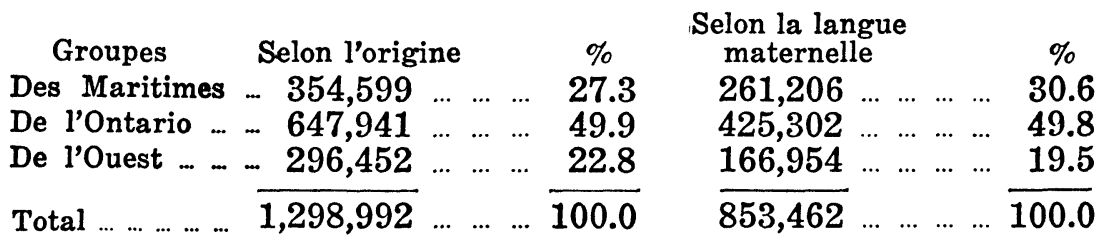

Le groupe franco-ontarien est aussi nombreux à lui seul que les deux autres groupes réunis, tant selon l'origine que selon la langue, et il conserve le même pourcentage dans un cas comme dans l'autre. Sur le plan de la langue maternelle, le groupe des Maritimes augmente son pourcentage, alors que celui de l'Ouest voit le sien diminuer, sans doute parce que l'assimilation a fait plus de ravages dans l'Ouest que dans les Maritimes.

\section{1 - La vie française aux Maritimes}

Des quatre provinces Maritimes, deux seulement sont entrées dans la Confédération en 1867, donc il y a un siècle: le NouveauBrunswick et la Nouvelle-Écosse. La troisième, l'Ile-du-PrinceÉdouard a attendu jusqu'en 1873, et la quatrième, Terre-Neuve, jusqu'en 1949. J'ai déjà dit qu'à l'époque de la Confédération il devait exister en cette région environ 85,000 habitants d'origine française, dont la langue maternelle devait être, pour la très grande majorité, le françaiis. Aujourd'hui, c'est-à-dire en 1961, la même région compte 354,599 habitants d'origine française et 261,206 de langue maternelle française. Cela signifie que la population d'origine française s'est multipliée par quatre, et la population de langue française, par trois, en moins d'un siècle.

Les difficultés faites à la vie française, comme nous le verrons, ont été grandes. Je me contente de citer ici la plainte que faisaient entendre devant la Commission Rowell-Sirois, en 1937, les Acadiens et les Canadiens français des Maritimes: 
Ironie du sort, les minorités des provinces Maritimes se trouvent aujourd'hui dans une situation moins favorable qu'en 1867. A cause de l'insuffisance des articles 93 et 133 , ou plutôt à cause de l'interprétation étroite qu'on leur a donnée, les minorités françaises et catholiques ont souffert énormément dans leur vie nationale et religieuse. Elles ont subi et subissent tous les jours de nouvelles pertes. Elles en subiront nécessairement de nouvelles, si des mesures ne sont prises pour faire respecter l'esprit sinon la lettre du pacte fédératif ${ }^{\mathbf{1 2}}$.

Depuis cette date, nous le verrons, la situation s'est améliorée dans certaines provinces, mais pas dans toutes.

\section{A Terre-Neuve}

Terre-Neuve présente cette double distinction de ne compter que dix-huit ans de Confédération et de posséder la plus petite minorité française de toutes les provinces canadiennes. Au recensement provincial de 1945 , la population d'origine française se chiffrait à 9,083; au premier recensement fédéral de 1951, elle s'élevait à 9,841 , mais elle allait presque doubler au cours des dix ans à venir, puisque le recensement de 1961 y dénombrait 17,191 citoyens d'origine française.

Malheureusement, de ce nombre, 14,634 , soit 85.2 p. cent, se sont déclarés de langue maternelle anglaise et seulement 2,522, soit 14.6 p. cent, de langue maternelle française, alors que 14,024 , soit $81.6 \mathrm{p}$. cent, ont dit ne savoir que l'anglais. Nous nous trouvons donc en face d'une population d'origine française anglicisée à plus de 80 p. cent. De cette situation la Confédération n'est certes pas responsable; il semble, au contraire, que l'entrée de Terre-Neuve dans la fédération canadienne ait sensibilisé davantage la province à l'existence et à la condition tout à fait spéciale de sa minorité française, mais pas au point de lui venir substantiellement en aide.

Tous les témoignages concordent pour démontrer que même la survivance de cette minorité est gravement compromise.

12 "Mémoire des Acadiens et des Canadiens français des provinces Maritimes à la Commission Rowell-Sirois", 1937, loc. cit., 1. 
Décrivant la pénible condition des Acadiens aux Maritimes, M. Emery LeBlanc ajoutait au passage: "La situation est encore plus inquiétante à Terre-Neuve, où il n'y a eu ni école française ni curé français depuis plus d'un demi-siècle." 13 Dans le Répertoire des Institutions canadiennes d'enseignement français, publié par l'ACELF, on lit cette description navrante de l'état du français à Terre-Neuve:

Les gens les plus âgés sont bilingues mais parlent le français dans l'intimité de leurs foyers. Les personnes d'âge moyen sont moins bilingues et font moins usage de la langue de leurs ancêtres, même dans leurs foyers. La nouvelle génération, c'est-àdire les garçons et les filles d'aujourd'hui, comprend le français mais hésite à le parler.

La situation semble avoir atteint un point où les gens d'origine française préfèrent, dans la plupart des cas, ne pas être identifiés comme étant différents de la majorité ... Il n'y a pas de professeurs de langue française ni d'écoles françaises à Terre-Neuve. Le français est enseigné comme matière régulière dans plusieurs écoles, mais il n'est pas obligatoire ... Malheureusement, le français est pauvrement enseigné dans les écoles de Terre-Neuve, ce qui est dû au fait que les maîtres ne l'emploient pas comme langue parlée ${ }^{14}$.

Même son de cloche de la part d'un jeune ethnologue de l'Université de Moncton. A la suite d'études et de recherches sur la culture française à Terre-Neuve, M. Jean-Claude Dupont n'a pu cacher son profond pessimisme au sujet de l'avenir du français dans cette province. Il ne reste plus à Terre-Neuve, a-t-il déclaré tout récemment, qu'environ 2,000 personnes à parler le français, mais il s'agit d'un français "baragouiné" qui n'a plus d'attrait pour les jeunes. La jeunesse ne veut pour rien au monde apprendre une langue qui a fait de leurs parents des inférieur au point de vue économique, culturel, social, etc. De la culture française il ne reste que des vestiges ${ }^{15}$.

13 Emery LeBlanc, Les Acadiens (Montréal, 1963), 121.

14 Répertoire des Institutions canadiennes d'enseignement frangais, 1961-1962 (Québec, 1962), 1165.

15 Jean-Claude Dupont, "La culture française en voie de disparition à Terre-Neuve?", dans L'Evangéline, 28 février 1967. 
Encore une fois, la Confédération comme telle ne peut être tenue responsable de la décadence du français à Terre-Neuve, mais il faut bien rappeler que, lors des accords de 1948-1949 qui ont abouti à l'entrée de Terre-Neuve dans la Fédération canadienne, personne, semble-t-il, ne s'est soucié de procurer à la minorité française d'autre protection que celle de l'article 133. Cela voulait dire en pratique que tout était laissé à la bonne volonté et à la bienveillance de la majorité et du gouvernement de la province, et que la minorité française allait, après comme avant la Confédération, continuer sa marche vers l'assimilation totale.

\section{Dans l'Ile-du-Prince-Edouard}

L'Île-du-Prince-Edouard a attendu six ans, c'est-à-dire jusqu'en 1873, pour faire son entrée dans la Confédération canadienne. Elle comptait alors près d'une dizaine de mille habitants d'origine française, des Acadiens pour la plupart. Il ne semble pas qu'on ait demandé ni qu'on ait accordé à ces derniers des garanties spéciales pour leur vie française. Munis de la seule protection fournie par les articles 133 et 93 de la Constitution de 1867, les Acadiens de l'île commencèrent leur vie en tant que citoyens de la nouvelle fédération. Quatre ans plus tard, c'était la tragédie.

En 1877, en effet, une loi du parlement de Charlottetown établissait un système scolaire dans lequel l'école était à la fois unique, obligatoire, non confessionnelle et, par voie de conséquence, de langue anglaise. Des démarches entreprises pour faire, sinon abolir, du moins adoucir cette loi ne donnèrent aucun résultat, pas plus à Ottawa qu'à Charlottetown. La vie des Acadiens de l'île va désormais se passer à arracher quelques concessions destinées à adoucir l'application de la loi et à permettre une certaine survie du français.

$\mathrm{Au}$ premier recensement fédéral tenu après l'entrée de l'île dans la Confédération, c'est-à-dire en 1881, leur nombre s'élevait à 10,751. Il n'était encore que de 11,971 quarante ans plus tard, soit en 1921. Encore quarante ans, et ce nombre, en 1961, attein- 
dra 17,418 sur une population totale de 104,629. Durant tout ce temps, c'est-à-dire de 1881 à 1961 , le pourcentage de la population d'origine française n'a cessé d'augmenter, passant de 9.9 à 16.64.

De ce point de vue de l'origine ethnique, l'évolution peut paraître assez satisfaisante, mais si on se place sur le plan de la langue maternelle, elle touche à la tragédie, comme l'indique le tableau suivant.

TABLEAU 8

Evolution de la population française dans l'Ile-du-Prince-Edouard

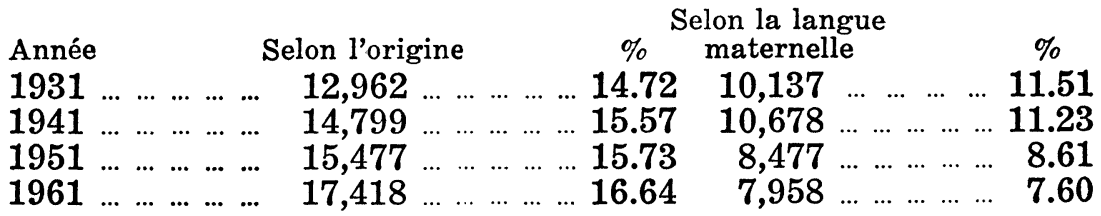

En ces trente dernières années, la population d'origine française a augmenté de 4,456, mais la population de langue maternelle française a diminué de 2,179 et ne forme plus que 7.60 p. cent de la population totale de l'île. Depuis 1941 , le nombre de personnes de langue maternelle française va sans cesse diminuant, il est passé de 10,678 à 7,958 au dernier recensement.

Un autre tableau aidera à saisir le tragique de la situation du groupe français dans l'île-du-Prince-Édouard.

\section{TABLEAU 9}

Bilan de la situation linguistique de la population d'origine française dans l'Île-du-Prince-Édouard, en 1961

D'origine française

Sachant l'anglais

De langue maternelle anglaise

Ne sachant que l'anglais

Sachant le français

De langue maternelle française ...

L'anglais et le français

$\mathrm{Ne}$ sachant que le français
Nombre

17,418

16,183

9,612

9,040

8,321

7,744

7,143

1,178
Pourcentage $\%$ 100.0 92.9

55.2

51.9

47.9

44.5

41.0

6.8 
Plus de la moitié de cette population d'origine française ne sait plus que l'anglais, et le reste, semble-t-il, s'anglicise à vive allure. En 1941, par exemple, la proportion des Canadiens d'origine française à se déclarer de langue maternelle anglaise n'était que de $29.1 \mathrm{p}$. cent; en 1961, cette même proportion s'élevait à 55.2 p. cent.

Peut-on parler d'un siècle de vie française dans l'île-duPrince-Edouard? Peut-être, mais quelle pauvre vie française ! Une vie sans cadres pour l'appuyer, sans institutions scolaires ni culturelles, une vie cantonnée à la famille et, en quelques rares endroits, à l'école et à la paroisse, bref une vie qui se détériore et s'use chaque jour davantage et dont les porteurs vont se raréfiant, voilà comment pourrait se caractériser la vie française dans l'île, voilà la vie que la majorité a faite à sa minorité dont la langue est pourtant l'une des deux langues officielles du Canada.

\section{En Nouvelle-Écosse}

Avec la Nouvelle-Écosse, qui fut l'une des provinces fondatrices de la Confédération, nous pouvons vraiment parler d'un siècle de vie fédérative, 1867-1967; mais ce siècle en a-t-il été un de vie française ? Il faudrait posséder une forte dose d'optimisme et refuser de voir la réalité pour l'affirmer sans réserve.

Dès 1864, quelques mois avant les grandes conférences de Charlottetown et de Québec, une loi impose en Nouvelle-Écosse un programme unique à toutes les écoles publiques, programme entièrement anglais. L'année suivante, cette loi se renforce de la cotisation obligatoire. Doublement atteintes, les écoles confessionnelles françaises doivent fermer leurs portes. En 1901, les Acadiens réussissent à obtenir un peu de français à l'école primaire; en 1940, un nouveau programme fait une part plus large au français. Aujourd'hui, il n'existe pas d'écoles séparées, mais seulement des écoles "bilingues" dans les districts scolaires habités par une population de langue française ${ }^{16}$. Le ministère

16 "Au point de vue scolaire, la loi ne reconnaît pas d'écoles séparées. Cela veut dire qu'officiellement toutes les écoles subventionnées par l'Etat sont neutres au point de vue religieux et anglaises au point de vue langue 
de l'Éducation accepte d'y reconnaître l'enseignement du français et le rend même obligatoire jusqu'en $9 \mathrm{e}$ année, mais les Acadiens, même s'ils possèdent un collège classique à Pointe-à-l'Église, n'ont pas d'école normale secondaire pour la préparation de professeurs de français.

Cette lutte d'un siècle qu'a dû livrer pour la survivance de ses écoles la minorité acadienne en Nouvelle-Écosse se reflète dans les statistiques démographiques. Au moment de la Confédération, la province comptait environ une trentaine de mille habitants d'origine française, formant $8 \mathrm{p}$. cent de la population totale. Aujourd'hui, c'est-à-dire en 1961, la même province en compte 87,883 , qui constituent $12 \mathrm{p}$. cent de l'ensemble des citoyens néo-écossais. La population d'origine française, qui était de 32,833 en 1871, s'y est donc multipliée presque par trois.

A l'exception de la période allant de 1911 à 1931, la population d'origine française a lentement progressé de dix ans en dix ans. En a-t-il été ainsi de la population de langue maternelle française ? Les résultats, ici, sont beaucoup moins encourageants.

\section{TABLEAU 10}

Évolution de la population française en Nouvelle-Écosse, de 1931 à 1961

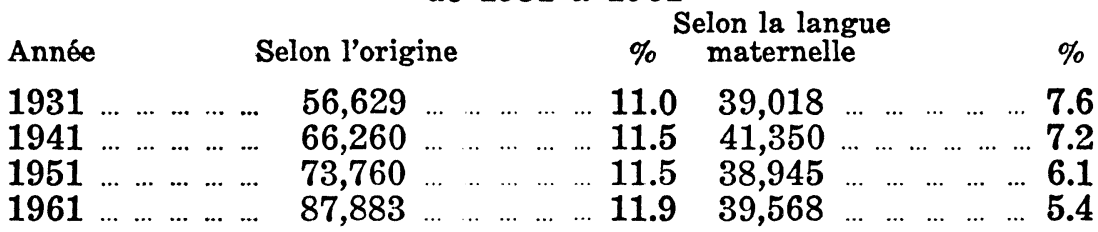

La population de langue maternelle française demeure stationnaire depuis 30 ans, même si la population d'origine française a augmenté de plus de 30,000 . Bien plus, on pourrait

d'enseignement. La pratique diffère cependant sensiblement de la loi. Dans les écoles acadiennes, le département tolère que la langue française soit la langue d'enseignement pour toutes les matières, sauf l'arithmétique et l'anglais. Les deux langues sont employées pour l'enseignement jusqu'à la $9 \mathrm{e}$ année. L'anglais devient ensuite la seule langue d'enseignement, sauf pour la grammaire française, en $10 \mathrm{e}$ et $11 \mathrm{e}$ années" (Paul-Emile Gosselin, L'empire français d'Amérique (Québec, 1943), 40.) 
soutenir que cette population de langue française d'aujourd'hui n'est guère plus nombreuse que celle qui existait, il y a un siècle, au moment où la Nouvelle-Écosse entrait dans la Confédération. Est-il besoin de souligner, en outre, que le pourcentage détenu par les gens de langue française par rapport à l'ensemble de la population va sans cesse diminuant ? En 1931, il était de 7.6, mais il n'est plus que de 5.4 en 1961.

Le tableau suivant résume la situation linguistique actuelle.

TABLEAU 11

Bilan de la situation linguistique de la population d'origine française en Nouvelle-Écosse, en 1961

Nombre

D'origine française

Sachant l'anglais

De langue maternelle anglaise ...

Ne sachant que l'anglais

Sachant le français

De langue maternelle française ...

L'anglais et le français

$\mathrm{Ne}$ sachant que le français
87,883

82,133

50,049

46,229

41,500

37,621

35,904

5,596 $\underset{\%}{\text { Pourcentage }}$

100.0

93.4

56.9

52.6

47.2

42.8

40.9

6.4

Nous sommes en face d'une minorité dont plus de la moitié des membres ne savent que l'anglais $(46,229$ sur 87,883$)$ et dont les deux cinquièmes seulement se disent encore de langue maternelle française. Le plus grave, cependant, est l'allure rapide prise par l'anglicisation. Sur 100 Canadiens d'origine française en Nouvelle-Écosse, il n'y en a plus que 47 à connaître le français, alors qu'en 1931 on en comptait 71, que 42 de langue maternelle française, alors qu'en 1931 on en comptait 67; de même, il y en a 52 qui ne savent que l'anglais, alors qu'en 1931 il n'y en avait que 28 .

On saisit alors dans quel sens l'on peut parler d'un siècle de vie française en Nouvelle-Écosse. Il s'agit d'une vie d'une grande faiblesse, qui aurait besoin, pour simplement durer, de la protection et de l'assistance de l'État provincial, d'une protection et d'une assistance beaucoup plus grandes en tout cas que celles 
qu'a constamment reçues la minorité anglaise au Québec depuis 1867. Les lui accordera-t-on avant qu'il ne soit trop tard ?

\section{Au Nouveau-Brunswick}

Le Nouveau-Brunswick, tout comme la Nouvelle-Écosse, fut l'une des premières provinces à s'associer en 1864-1867 pour former la Confédération canadienne. Il comptait alors une quarantaine de mille habitants d'origine française, soit $16 \mathrm{p}$. cent de sa population totale. Personne, semble-t-il, ne songea à leur procurer une protection spéciale, en plus des garanties offertes par les articles 133 et 93.

Quatre ans plus tard, en 1871, une loi provinciale balayait les écoles catholiques et françaises; la lutte pour la survivance commençait et allait se poursuivre sans arrêt jusqu'à la remontée spectaculaire dont nous sommes aujourd'hui les témoins ${ }^{17}$.

En 1871, la population d'origine française s'élève à 44,907; trente ans plus tard, elle est de 80,097; encore trente ans, et elle atteindra 136,999 (en 1931), et enfin 232,127 au dernier recensement de 1961, formant aujourd'hui $38.8 \mathrm{p}$. cent de la population totale. Les Acadiens se sont ainsi multipliés par cinq depuis la Confédération, doublant leurs effectifs tous les quarante ans.

Chose étonnante! Les pertes sur le plan de la langue sont relativement peu considérables, beaucoup moins considérables en tout cas que dans les trois autres provinces. Si, durant longtemps, les Acadiens ont vécu repliés sur eux-mêmes et sans trop d'instruction, ils ont tenu farouchement à leur langue et ne se sont pas laissés angliciser. Voici sur ce sujet quelques statistiques intéressantes.

17 On pourra lire dans l'ouvrage de l'abbé Lionel Groulx, L'enseignement français au Canada, II - Les écoles des minorités, 27-54, le récit des luttes scolaires au Nouveau-Brunswick. En conclusion, l'auteur écrit: “Après de tels événements, se pouvait-il que l'égalité des races et des croyances devant la constitution ne parût un mythe et l'alliance de 1867 , un marché de dupes? Il n'est pas excessif d'écrire qu'entre ces années 1871 et 1875, il s'était commis au Canada l'une des plus lourdes, l'une des plus fatales erreurs politiques. Dès lors, en effet, ce rêve absurde s'est formé de fonder une nation canadienne sur l'inégalité juridique et politique des races, autant dire sur la discorde perpétuelle (54)." 
TABLEAU 12

Evolution de la population française au Nouveau-Brunswick, de 1931 à 1961

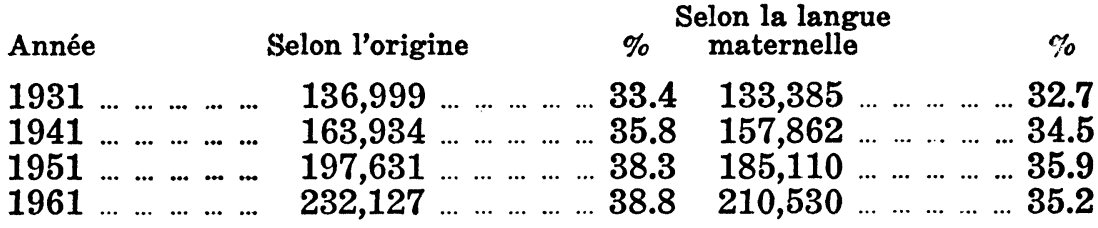

Pour diverses raisons, la dernière décennie n'a pas donné aux Acadiens du Nouveau-Brunswick l'augmentation qu'ils avaient droit d'espérer; même leur pourcentage par rapport à l'ensemble de la population, pourcentage fondé sur la langue maternelle française, a légèrement diminué de 1951 à 1961. Il n'en reste pas moins que la proportion des anglicisés demeure très basse parmi eux.

\section{TABLEAU 13}

Bilan de la situation linguistique de la population d'origine française au Nouveau-Brunswick, en 1961

Nombre

D'origine française

Sachant le français

De langue maternelle française ..

Sachant l'anglais

$\mathrm{Ne}$ sachant que le français

$L$ 'anglais et le français

De langue maternelle anglaise .

$\mathrm{Ne}$ sachant que l'anglais
232,127

208,440

203,258

122,397

109,282

99,158

28,226

23,239
Pourcentage
$\%$

100.0

89.8

87.6

52.7

47.1

42.7

12.2

10.0

Quand on songe aux difficultés que les Acadiens ont affrontées au cours du dernier siècle pour maintenir leur vie française, on ne peut qu'être rempli d'admiration en considérant de pareils résultats: près de $90 \mathrm{p}$. cent des Acadiens savent le français et près de $88 \mathrm{p}$. cent sont encore de langue maternelle française !

Ces résultats sont d'autant plus encourageants qu'ils correspondent à une véritable remontée des Acadiens dans la plupart 
des domaines de la vie publique, scolaire, culturelle et économique. L'un des leurs est premier ministre de la province, la traduction simultanée va être installée au parlement de Fredericton et il s'en est fallu de peu que leur langue ne fût proclamée langue officielle de la province à la fin de mars 1967.

Bref, on peut parler maintenant de vie française au Nouveau-Brunswick. C'est une vie qui s'organise autour de nombreuses écoles, de collèges, voire d'une université: celle de Moncton, et qui peut compter sur l'appui d'institutions culturelles appelées à se développer dans l'avenir: un quotidien, des postes de radio et de télévision, des associations de toutes sortes. Longtemps, la vie française au Nouveau-Brunswick n'a été qu'un faible feu couvant sous la cendre; c'est aujourd'hui une force avec laquelle il faut compter et une flamme dont le rayonnement grandit chaque jour davantage ${ }^{18}$.

\section{2 - La vie française en Ontario}

De toutes les provinces canadiennes, l'Ontario est celle qui a toujours été mêlée de plus près à la vie de la province de Québec et chez qui la vie française de cette dernière a toujours eu tendance à déborder. Aujourd'hui comme au moment de la Confédération, l'Ontario renferme sur son sol environ la moitié de la population d'origine française vivant en dehors du Québec. C'est dans cette province que les Canadiens français se sont développés le plus rapidement depuis 1867 , passant de 75,000 à plus de 600,000. En 1871, lors du premier recensement fédéral, ils étaient en effet 75,383 et formaient $4.6 \mathrm{p}$. cent de la population totale; en 1961, ils sont 647,941 et forment $10.4 \mathrm{p}$. cent de la population ontarienne.

18 Le Répertoire des Institutions d'enseignement français résume ainsi la situation du français au Nouveau-Brunswick en 1961-1962: "On compte dans la province 124 paroisses canadiennes-françaises. Dans les écoles "bilingues", le français est reconnu comme matière d'enseignement scolaire par le Département d'Education. Dans ces mêmes écoles, l'enseignement du francais est obligatoire. Des inspecteurs qualifiés dans ce domaine sont à l'emploi du Département d'Education. Malheureusement, le personnel enseignant n'est pas assez nombreux pour satisfaire aux demandes. En général, les titulaires de classes sont bien préparés pour l'enseignement. Il n'existe pas d'école normale secondaire pour la préparation des professeurs de français. Le français n'est d'ailleurs pas reconnu comme langue officielle nécessaire dans l'exercice des fonctions administratives et publiques (960)." 
Les Franco-Ontariens ont toujours tenu à conserver le français dans leurs écoles. Leur détermination apparut au grand jour lors des luttes qu'ils durent soutenir, de 1913 à 1927, contre le fameux Règlement 17, dont le but véritable était de "restreindre l'usage du français dans les écoles bilingues" 19. A cette occasion, la province de Québec tout entière, par ses chefs tant religieux que politiques, par ses journaux et ses écrivains, accorda son appui à la cause de la minorité. Le Règlement 17 finit par être rappelé, et les Franco-Ontariens s'acharnèrent à refaire leurs écoles et leurs collèges. Tout récemment, ils faisaient un pas audacieux en décidant de réclamer du gouvernement de la province l'établissement d'un réseau d'écoles secondaires bilingues ou françaises intégré au système scolaire public non confessionnel ${ }^{20}$.

Il était temps que le gouvernement vienne en aide aux Franco-Ontariens, car l'anglicisation exerce de terribles ravages dans leurs rangs, comme le montre le tableau suivant.

TABLEAU 14

Evolution de la population française en Ontario, de 1931 à 1961

\begin{tabular}{|c|c|c|c|}
\hline & & & \\
\hline & Selo & & \\
\hline $\begin{array}{lllll}\ldots & \ldots & \ldots & \ldots \\
\ldots & \ldots & \ldots & \ldots\end{array}$ & $\begin{array}{llllll}299,732 & \ldots & \ldots & \ldots & \ldots & \ldots \\
373,990 & \ldots & \ldots & \ldots & \ldots & \ldots \\
477,677 & \ldots & \ldots & \ldots & \ldots & \ldots \\
647,941 & \ldots & \ldots & \ldots & \ldots & \ldots\end{array}$ & $\begin{array}{r}9.9 \\
10.4 \\
10.4\end{array}$ & $\begin{array}{lllllll}236,386 & \ldots & \ldots & \ldots & \ldots & \ldots & \mathbf{6} . \\
289,146 & \ldots & \ldots & \ldots & \ldots & \ldots & \mathbf{7} \\
28 & \\
341,502 & \ldots & \ldots & \ldots & \ldots & \ldots & \mathbf{7} . \\
425,302 & \ldots & \ldots & \ldots & \ldots & \ldots & \mathbf{6} .\end{array}$ \\
\hline
\end{tabular}

De 1951 à 1961, les Franco-Ontariens d'origine ont maintenu leur pourcentage dans la province, mais depuis 1941 leur pourcentage fondé sur la langue maternelle française va sans cesse diminuant, de peu il est vrai, mais la tendance paraît constante.

19 Voir le récit de ces luttes dans l'ouvrage de l'abbé Lionel Groulx, L'enseignement français au Canada, II - Les écoles des minorités, 206-239. Voir aussi la conférence prononcée, le 23 novembre 1966, à Montréal, par le premier ministre de l'Ontario, M. John Robarts (Texte français dans le Devoir du 29 novembre 1966).

20 Voir à ce sujet le Devoir du 20 février 1967 et du 30 mars 1967: "Mgr Plourde appuie le projet d'associer les écoles catholiques au secteur public." 
Il faut en rechercher la cause dans le nombre croissant d'anglicisés, c'est-à-dire de Franco-Ontariens qui ne savent plus que l'anglais, que compte la minorité franco-ontarienne. En 1931, elle en comptait 20 p. cent, en 1941, 24 p. cent, en 1951, 28 p. cent, et en 1961, 33.6 p. cent, soit 217,751. Aujourd'hui, la situation générale se présente ainsi sur le plan linguistique.

TABLEAU 15

Bilan de la situation linguistique de la population d'origine française en Ontario, en 1961

\begin{tabular}{|c|c|c|}
\hline & Nombre & $\begin{array}{c}\text { Pourcentage } \\
\%\end{array}$ \\
\hline origine française & 647,941 & 100.0 \\
\hline Sachant l'anglais & 556,204 & 85.8 \\
\hline Sachant le français & 427,527 & 66.0 \\
\hline De langue maternelle française & 397,728 & 61.4 \\
\hline L'anglais et le français & 338,453 & 52.2 \\
\hline De langue maternelle anglaise & 244,566 & 37.7 \\
\hline Ne sachant que l'anglais & 217,751 & 33.6 \\
\hline Ne sachant que le français & 89,074 & 13.7 \\
\hline
\end{tabular}

La minorité franco-ontarienne sait plus l'anglais que le français ( $85.8 \%$ contre $66 \%$ ) et elle compte beaucoup plus de gens qui ne savent que l'anglais que de personnes qui ne savent que le français $(33.6 \%$ contre $13.7 \%$ ) ; par contre, 61.4 p. cent de ses membres se déclarent de langue maternelle française et 33.6 p. cent, de langue maternelle anglaise.

En ces dernières années, la vie française semble vouloir prendre un nouveau départ en Ontario; les difficultés, certes, demeurent fort grandes ${ }^{21}$, mais depuis un siècle jamais un

21 Dans le Mémoire soumis, en 1964, à la Commission royale d'enquête sur le bilinguisme et le biculturalisme, l'Association canadienne-française d'Education d'Ontario déclare: "Ce que nous réclamons pour les minorités françaises à travers tout le Carada comme pour la minorité française de l'Ontario, c'est le même traitement que reçoivent les minorités de langue anglaise dans la province de Québec et plus spécialement: 1) que le français soit reconnu langue première et l'anglais, langue seconde pour les FrancoOntariens; 2) que tout l'enseignement se donne en français, du primaire au supérieur, sauf bien entendu, l'enseignement de l'anglais; 3) que l'on ne tente pas d'imposer une langue seconde à nos enfants avant qu'ils aient acquis une maîtrise suffisante de leur langue; 4) la distribution per capita 
gouvernement ontarien ne lui avait manifesté autant de sympathie que le gouvernement actuel. On ne peut que souhaiter que cette sympathie se traduise par des actes qui rendent pleine justice à la si méritante minorité franco-ontarienne.

\section{3 - La vie française dans l'Ouest canadien}

Au moment de la Confédération, sur les territoires aujourd'hui occupés par les quatre provinces du Manitoba, de la Saskatchewan, de l'Alberta et de la Colombie-Britannique, il pouvait y avoir une douzaine de mille habitants d'origine française, dont près de la moitié dans le seul Manitoba. Un siècle plus tard, en 1961, ces quatre provinces comptent 294,049 citoyens d'origine française, formant $6.7 \mathrm{p}$. cent de leur population, mais seulement 165,517 habitants de langue maternelle française, ne formant plus que $3.44 \mathrm{p}$. cent de l'ensemble. Le progrès a donc été considérable en un siècle, mais les pertes aussi, lesquelles se montent à environ $40 \mathrm{p}$. cent de toute la population d'origine française. Cette dernière y occupe une situation extrêmement difficile et elle n'y détient même que le troisième ou le quatrième rang en importance numérique.

\section{Au Manitoba}

Le Manitoba est entré dans la Confédération en 1870 avec des garanties spéciales pour sa minorité catholique et française, garanties inscrites dans sa propre constitution, laquelle sera à son tour confirmée en 1871 par un acte du Parlement impérial. L'article 22 de l'Acte du Manitoba défendait à la législature provinciale de porter atteinte "à aucun droit ou privilège conféré, lors de l'Union, par la loi ou par la coutume" aux écoles séparées. L'article 23 du même Acte établissait dans la province le même bilinguisme officiel qui existait alors au Québec, le français et l'anglais étant mis sur un pied d'égalité.

de tous les argents recueillis pour fins scolaires ou destinés à des fins scolaires ou académiques, $y$ compris les montants destinés au niveau universitaire; 5) l'établissement d'une surintendance des écoles françaises qui aurait pleine autorité pour administrer toutes nos écoles et tout notre système d'enseignement" (Ottawa, 1964), 12. 
On sait ce qu'il advint de ces garanties. Vingt ans plus tard, en 1890, c'était l'attaque brutale contre l'école confessionnelle et la langue française, l'abolition de la première et les restrictions imposées à la seconde, en attendant son interdiction totale dans les écoles en 1916. Inutile de refaire ici le récit de cette douloureuse histoire par laquelle est passée la minorité française avant de se convaincre qu'il n'y avait pour elle rien à attendre des autorités officielles, pas plus de celles de Londres, d'Ottawa que de celles de Winnipeg, et que la seule chose à faire était de prendre courageusement son destin en main, de fonder et de gérer son propre système d'éducation ${ }^{22}$.

Les Franco-Manitobains sont passés de cinq à six mille qu'ils étaient en 1870 à 83,936 en 1961 ; ils forment 9.1 p. cent de la population totale. Malheureusement, l'hostilité du milieu n'a point favorisé chez eux la conservation de leur langue maternelle, comme en témoigne le tableau suivant.

\section{TABLEAU 16}

Evolution de la population française au Manitoba, de 1931 à 1961

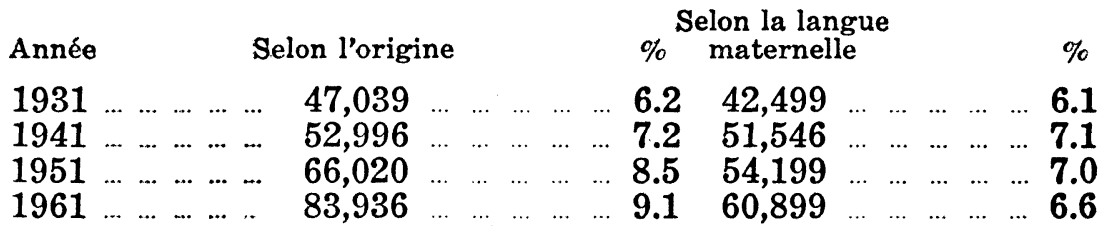

En dépit de leurs progrès selon l'origine, les Franco-Manitobains, considérés du point de vue de leur langue maternelle, perdent du terrain dans la province depuis 1941, leur pourcentage passant de 7.1 à 6.6. Leur situation générale est la suivante.

${ }^{22}$ L'abbé Lionel Groulx, dans son ouvrage L'enseignement français au Canada, II - Les écoles des minorités, a raconté cette histoire émouvante, 71-137. 


\section{TABLEAU 17}

Bilan de la situation linguistique de la population d'origine française au Manitoba, en 1961

\begin{tabular}{|c|c|c|}
\hline & Nombre & $\begin{array}{c}\text { Pourcentage } \\
\%\end{array}$ \\
\hline D'origine française & 83,936 & 100.0 \\
\hline Sachant l'anglais. & 76,285 & 90.9 \\
\hline Sachant le francais & 59,081 & 70.4 \\
\hline De langue maternelle française & 56,433 & 67.2 \\
\hline L'anglais et le français ... ............ & 51,860 & 61.7 \\
\hline De langue maternelle anglaise & 25,451 & 30.3 \\
\hline Ne sachant que l'anglais ... ... ... & 24,425 & 29.1 \\
\hline Ne sachant que le français ... & 7,221 & 8.6 \\
\hline
\end{tabular}

Les Franco-Manitobains savent beaucoup plus l'anglais que le français, mais ils gardent encore leur préférence au français comme langue maternelle. Il faut cependant déplorer que 24,425 d'entre eux, soit près de $30 \mathrm{p}$. cent, déclarent ne savoir que l'anglais.

Tout comme au Nouveau-Brunswick et en Ontario, le français jouit, en cette année 1967, d'un climat de plus grande sympathie au Manitoba. En mars 1967, le ministre G. Johnson présentait un projet de loi ayant pour but de permettre l'usage du français comme langue d'enseignement, une demi-journée par jour, dans les écoles publiques de la province. A cette occasion, il déclarait: "Il est très convenable en cette année du centenaire de la Confédération que le français, une des deux langues mentionnées dans l'Acte de l'Amérique du Nord britannique en 1867, soit réintégré comme langue d'enseignement dans nos écoles publiques ${ }^{23}$." Il faut souhaiter que ce projet réparateur n'arrive pas trop tard pour arrêter la marche à l'anglicisation dont le rythme s'est fait plus rapide en ces dernières années.

${ }^{23}$ Voir, à ce sujet, La Liberté et le Patriote, 30 mars 1967, éditorial, p. 2; aussi le Devoir, du 23 mars 1967, éditorial de Vincent Prince: "Le français fait un gain substantiel au Manitoba"; et la série d'articles de Marcel Gingras publiés dans le Droit, à partir du 27 mars: "Le français au Canada"; les articles 3 à 6 de cette série portent sur la situation de l'école française au Manitoba. 


\section{En Saskatchewan}

Devenus possession canadienne dès 1869 , les Territoires du Nord-Ouest ne reçurent leur première organisation politique qu'en 1875; deux ans plus tard, l'article 110 de la Constitution des Territoires reproduisait la substance de l'article 133 de l'Acte de l'Amérique du Nord britannique: "Toute personne pourra faire usage soit de la langue française, soit de la langue anglaise dans les débats du Conseil ou de l'Assemblée législative des Territoires, ainsi que dans les procédures devant les Cours..."

C'était trop beau pour durer bien longtemps. Dès 1890 , l'Assemblée de Regina réclame du parlement fédéral l'abrogation de cet article 110, permission qui lui est accordée l'année suivante. En 1892, une Ordonnance fait de l'anglais la seule langue possible d'enseignement, sauf au cours primaire. Lors de la création des deux provinces de la Saskatchewan et de l'Alberta, en dépit des protestations de Bourassa, aucune garantie spéciale n'est reconnue aux minorités catholiques et françaises. A partir de 1929, tout doit s'enseigner en anglais à l'école. Écrivant en 1937, M. l'abbé Maurice Baudoux disait à ce sujet: "Pour le pouvoir provincial, le français ne compte absolument pas dans le domaine législatif, judiciaire ou administratif: tout y est uniquement en anglais ... L'anglais est la seule langue d'enseignement à tous les degrés et pour toutes les matières enseignées durant les heures de classe, y compris le français et le catéchisme, par conséquent..." 24

Heureusement, en cette année 1967, la situation paraît s'améliorer. Le parlement de Regina a voté, en mars, une loi permettant aux commissions scolaires locales d'autoriser l'enseignement en français pour une période d'une heure par jour, ainsi que l'enseignement de la religion, pendant une autre demi-heure quotidienne, dans une langue "autre que l'anglais".

Il était grand temps. Demeurée presque stationnaire depuis 1931, la population d'origine française a subi de très lourdes

\footnotetext{
24 Maurice Baudoux, "La situation juridique du français en Saskatchewan", Deuxième Congrès de la Langue française, 1937, Mémoires (Québec, 1938), II : 70-71.
} 
pertes du point de vue de sa langue maternelle, comme le montre le tableau suivant.

TABLEAU 18

Evolution de la population française en Saskatchewan, de 1931 à 1961

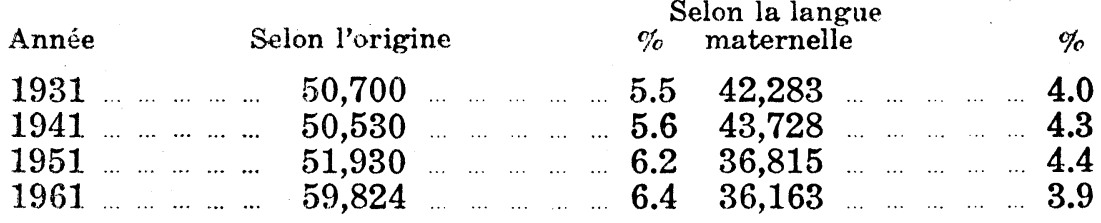

La population de langue maternelle française diminue depuis vingt ans; elle est passée de 43,000 à 36,000 et elle ne représente plus que $3.9 \mathrm{p}$. cent de la population totale. Les statistiques qui suivent donnent une idée de la gravité de la situation actuelle.

\section{TABLEAU 19}

Bilan de la situation linguistique de la population d'origine française en Saskatchewan, en 1961

Nombre

D'origine française

Sachant l'anglais

Sachant le français

De langue maternelle française

L'anglais et le français

De langue maternelle anglaise

$\mathrm{Ne}$ sachant que l'anglais

$\mathrm{Ne}$ sachant que le français
59,824

56,294

35,128

32,611

31,602

25,860

24,692

3,526
Pourcentage
$\%$

100.0

93.8

58.5

54.4

52.5

43.1

41.1

5.9

L'anglais est plus connu que le français. Le nombre de personnes de langue maternelle française dépasse encore celui des gens de langue maternelle anglaise, mais, par contre, 24,692 des Franco-Canadiens de la Saskatchewan, soit plus de 40 p. cent, ne savent plus que l'anglais. (En 1931, ce pourcentage n'était encore que de 16 et, en 1941, que de 31.5). Tel est le résultat de la pression hostile du milieu dans lequel ils doivent vivre leur vie française. 


\section{En Alberta}

En Alberta, l'évolution et la situation actuelle de la vie française ressemblent fort à ce que l'on vient de voir en Saskatchewan. Le français, reconnu langue officielle en 1877, y perd ses droits en 1892 et n'obtient aucune garantie spéciale lors de la création de la province en 1905. En 1911, il y a environ 20,000 Albertains d'origine française, en 1961 on en compte 83,319 , mais beaucoup n'ont pas conservé leur langue maternelle.

TABLEAU 20

Evolution de la population française en Alberta, de 1931 à 1961

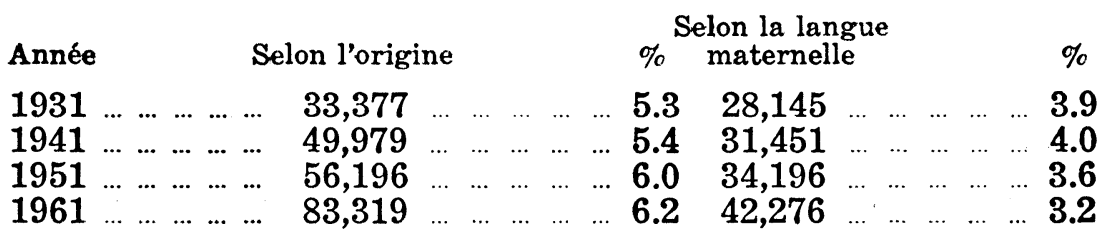

En trente ans, la population d'origine française a, non seulement maintenu, mais légèrement augmenté son pourcentage en Alberta, mais la population de langue maternelle française, elle, a vu le sien décroître constamment, par suite des gains massifs faits par l'anglicisation chez les Franco-Albertains.

TABLEAU 21

Bilan de la situation linguistique de la population d'origine française en Alberta, en 1961

Nombre

D'origine française

Sachant l'anglais

Sachant le français

De langue maternelle anglaise ...

Ne sachant que l'anglais

De langue maternelle française ..

L'anglais et le français

Ne sachant que le français
83,319

78,100

42,252

41,488

40,702

37,398

4,854
38,978 $\underset{\%}{\text { Pourcentage }}$

100.0

93.7

50.7

49.9

48.8

46.8

44.8

5.8 
La moitié seulement des Franco-Albertains savent encore le français et la moitié d'entre eux ont l'anglais pour langue maternelle; en outre, $48.8 \mathrm{p}$. cent ne savent plus que l'anglais. Tels sont les fruits de la politique qui, en 1892, supprimait les droits du français dans la province et qui a toujours, par la suite, mesuré au compte-gouttes les heures accordées à cette langue dans l'enseignement ${ }^{25}$.

\section{En Colombie-Britannique}

Bien que les Canadiens français aient été, depuis toujours, mêlés à la vie de la Colombie-Britannique, leur langue n'a jamais joui, en cette province, comme ce fut le cas dans les trois provinces des Prairies, de la reconnaissance officielle. Ils s'y développèrent, en outre, beaucoup plus lentement. En 1881, on n'y comptait encore que 916 habitants d'origine française, et seulement 11,246 en 1921. C'est après Terre-Neuve, la province où les ravages de l'anglicisation sont les plus élevés.

25 Dans le Répertoire des Institutions d'enseignement français, publié par l'ACELF en 1961-1962, on lit ce qui suit au sujet de l'enseignement du français en Alberta: "Le français est reconnu comme matière d'enseignement scolaire par le Département d'Education. Dans les écoles "bilingues", l'enseignement du français est facultatif. Il n'y a pas d'inspecteur qualifié dans ce domaine. Le Ministère d'Education reconnaît les "écoles séparées" de langue française. Le personnel enseignant de langue française est insuffisant... Il existe une école normale secondaire pour la formation de professeurs de français: le Collège d'Education de l'Université Laval au Collège Saint-Jean, à Edmonton. Le français n'est pas reconnu comme langue officielle nécessaire dans l'exercice des fonctions administratives et publiques (941)."

Sur l'évolution et la situation actuelle du fait français en Alberta, il faut lire l'important mémoire présenté en décembre 1965 à la Commission royale d'enquête sur le bilinguisme et le biculturalisme par l'Association canadienne-française de l'Alberta. Le texte en a été publié dans l'Almanach franco-albertain, de 1966. Deux extraits seulement: "En résumé, les lois qui garantissent l'enseignement religieux, malgré l'intention des législateurs de 1867 et de 1875, n'accordent aucun droit spécial à l'enseignement du français, l'anglais demeurant la seule langue d'enseignement reconnue. Et depuis 1892, le bilinguisme est proscrit à la législature et, semble-t-il, dans les cours de justice...

L'on peut dire que, d'une façon générale, les gens qui ne sont pas de même origine ethnique que nous acceptent très bien les Canadiens français comme individus, à condition cependant qu'ils parlent anglais et qu'ils n'affichent pas trop en groupe leur nationalisme. Cette acceptation se retrouve davantage dans la classe plus instruite ou chez nos concitoyens qui ont habité l'est du Canada. Quant à la masse de la population anglaise, elle ne se pose même pas le problème de l'acceptation" (Appendice à l'Almanach franco-albertain, 1966, 11-12). 
TABLEAU 22

Evolution de la population française en Colombie-Britannique, de 1931 à 1961

Année Selon l'origine

$$
\% \begin{gathered}
\text { Selon la langue } \\
\text { maternelle }
\end{gathered}
$$

1931 15,028

1941 21,876

1951 41,919

$2.2 \quad 7,768$

De 1951 à 1961, la population d'origine française a augmenté de 25,051, mais la population de langue maternelle française n'a augmenté que de 6,813; de plus, la première a augmenté son pourcentage dans la province, mais pas la seconde.

Le tableau suivant nous donne un aperçu de la situation tragique de cette minorité.

\section{TABLEAU 23}

Bilan de la situation linguistique de la population d'origine française en Colombie-Britannique, en 1961

D'origine française

Sachant l'anglais

De langue maternelle anglaise

$\mathrm{Ne}$ sachant que l'anglais

Sachant le français

L'anglais et le français

De langue maternelle française

Ne sachant que le français
Nombre

66,970

64,959

43,393

41,096

25,698

23,863

22,592

1,835
$\underset{\%}{\underset{\%}{P} \text { ourcentage }}$

100.0

97.0

64.8

61.4

38.3

35.6

33.7

2.8

Près des deux tiers de cette population sont de langue maternelle anglaise et plus de $60 \mathrm{p}$. cent ne savent plus que l'anglais. Des 66,970 Franco-Canadiens de Colombie il ne reste que 22,592, soit presque exactement le tiers, à se dire encore de langue maternelle française.

Est-il besoin de rappeler que le français n'a aucune existence officielle en cette province? On y compte cependant six parois- 
ses canadiennes-françaises, lesquelles s'efforcent tant bien que mal de maintenir des écoles dites "publiques" et d'y enseigner un peu de français. Mais jusqu'ici le gouvernement a refusé de reconnaître qu'il existait en Colombie un problème canadienfrançais, voire une minorité canadienne-française ${ }^{26}$.

Devant le tableau des pertes enregistrées par ses co-nationaux en dehors du Québec et des obstacles posés à la simple existence de la vie française dans les autres provinces depuis un siècle, il est difficile à un Canadien français du Québec de rester froid et indifférent, difficile de ne pas crier à l'injustice. Il voit, d'un côté, dans sa propre province, une minorité anglaise florissante et riche, à qui rien n'a été refusé et qui a toujours joui en paix de tous ses droits et privilèges, une minorité qui parle sa langue là et partout où elle le veut, qui possède ses écoles, ses universités, ses journaux, ses postes de radio et de télévision, une minorité qui, de dix ans en dix ans, affiche à chaque recensement fédéral les gains considérables qu'elle a effectués en faveur de sa langue chez les autres groupes ethniques du Québec: en 1961, par exemple, la population d'origine britannique dans la province s'élevait à 567,057 , et la population de langue maternelle anglaise à 697,402 , soit un surplus de 130,345. Ce même Canadien français du Québec contemple, d'un autre côté, le spectacle des siens livrés sans défense à l'assimilation progressive dans les autres provinces de la Confédération canadienne, des siens à qui on s'est empressé d'enlever leurs droits explicites là où ils en avaient et à qui on a refusé d'appliquer les garanties des articles 133 et 93 de la constitution canadienne, des siens qui, à cause de cette politique,

26 Déclaration du premier ministre de la Colombie-Britannique, W.A.C. Bennett, de passage à Montréal, en avril 1963 (cf. le Devoir, 6 avril 1963), et du procureur général de la même province, William Bonner, au cours d'une conférence de presse à l'occasion du congrès annuel de l'Association canadienne des Educateurs de langue française à Vancouver (cf. le Devoir, 26 août 1963). 
sont maintenant anglicisés pour plus de la moitié dans au moins quatre provinces sur neuf.

Comment, devant un tel spectacle, pourrait-il admettre que la Confédération s'est développée "d'après le principe de l'égalité des deux peuples qui l'ont fondée" ? Comment pourrait-il croire encore que le Canada tout entier est sa patrie, quand on a tout fait pour tuer en lui. le sentiment patriotique canadien? Il y avait un moyen d'enraciner et de faire croître pareil sentiment: permettre à la communauté canadienne-française de faire du Canada tout entier sa patrie, et cela en favorisant la vie française dans toutes et chacune des provinces. On a préféré faire le contraire et nier à cette communauté le droit de vivre en tant que telle, avec sa langue et ses écoles, en dehors du Québec.

Un siècle de vie françaïse en dehors du Québec ! Ce siècle aurait pu se dérouler d'une façon tellement différente si la majorité avait sincèrement et pleinement accepté l'association dans l'égalité des droits et des chances avec la minorité, par tout le pays, au lieu de bloquer cette dernière aux frontières du Québec et de lui dire: Hors de ces frontières vous n'êtes plus chez vous, vous n'avez plus rien et vous n'êtes plus rien en tant que communauté nationale; vous serez traités comme n'importe quelle autre minorité, comme n'importe quel autre groupe d'immigrants. Et c'est ainsi que des milliers et des centaines de milliers - près d'un demi-million - de FrancoCanadiens ont été assimilés et sont aujourd'hui perdus pour la vie française en dehors du Québec.

Le premier siècle de régime fédératif qui vient de s'écouler a été pour cette vie française hors du Québec un long et dur hiver; des signes récents laissent entrevoir un adoucissement du climat, un changement de température et l'arrivée possible du printemps. Si ces prévisions se réalisent, la persévérance opiniâtre à survivre des minorités françaises n'aura pas été vaine et peut-être alors leur sera-t-il permis de vivre un deuxième siècle de vie fédérative sous le triple signe de la paix, de la 
justice et de la fraternité, comme le demande la récente lettre collective de l'épiscopat du Canada à l'occasion du centenaire de la Confédération canadienne ${ }^{27}$.

RICHARD ARĖS, S.J.

27 "Les minorités vivant au Canada ne sont pas toutes dans la même situation. On doit veiller à assurer à chacune un traitement équitable et savoir accepter les sacrifices que ce traitement impose dans le coût de certains services, comme l'éducation, par exemple... Quand on réfléchit au problème des minorités au Canada, il faut aussi admettre qu'on peut à bon droit juger intolérable le régime politique qui, dans des situations analogues, n'assure pas le même traitement aux minorités de langue française qu'à celles de langue anglaise" (Ottawa, 7 avril 1967, 6-7).

En 1945, le même épiscopat avait déjà déclaré: "Nous tenons à proclamer bien haut que la législation scolaire de la plupart des provinces Nous semble appeler de profonds redressements, à l'endroit surtout de l'élément catholique et de l'élément français, si l'on veut qu'elle reflète vraiment l'esprit d'entente cordiale qui a présidé à la constitution canadienne. Aussi longtemps, en effet, que les inégalités flagrantes qui subsistent, sur ce terrain, entre le traitement qu'accorde la province de Québec à ses minorités religieuses et ethniques d'une part, et celui que leur imposent les autres provinces d'autre part, il Nous paraît vain de parler d'égalité d'avantages et de sacrifices, vain aussi d'espérer voir régner chez nous cette confiance mutuelle indispensable à la paix et à la prospérité publiques." 